\title{
Crise hipertensiva na odontologia
}

\section{Hypertensive crisis in dentistry}

Júllian Dalla Libera Pegoraro* Cristiane Aparecida de Oliveira ${ }^{* *}$

\section{Resumo}

Objetivo: o presente estudo tem como objetivo reunir informações sobre a crise hipertensiva, diferenciando as emergências hipertensivas das urgências hipertensivas, de modo a apresentar uma opção de conduta para cirurgiões-dentistas e colaborar para o melhor entendimento sobre o tema pelos profissionais ainda inseguros em emergências médicas. Revisão de literatura: caracterizada por um aumento rápido, intenso e inapropriado da pressão arterial, a crise hipertensiva é uma das complicações da hipertensão arterial. Essa crise pode ocorrer devido a um desequilíbrio entre o débito cardíaco e a resistência vascular periférica. $O$ aumento na pressão arterial sobrecarrega os vasos sanguíneos e o coração, requerendo rápida e correta correção, especialmente em pacientes com outras patologias. Crises hipertensivas podem ou não estar associadas a lesões de órgãos-alvo, diferenciando as emergências hipertensivas das urgências hipertensivas. Considerações finais: é imprescindível que o cirurgião-dentista saiba como proceder para prevenir uma crise hipertensiva, pois o atendimento odontológico está intimamente ligado ao medo, que promove um aumento da ansiedade e, por conseguinte, do estresse.

Palavras-chave: Hipertensão. Tratamento de emergência. Odontologia.

\section{Introdução}

Considerada como um dos fatores de risco cardiovascular mais importantes, a hipertensão arterial sistêmica é uma condição bastante prevalente na população adulta ${ }^{1}$, e atinge aproximadamente de $15 \%$ a $20 \%$ da população brasileira ${ }^{2}$.

Em muitos casos, a primeira forma de apresentação da hipertensão arterial sistêmica é a crise hipertensiva ${ }^{3}$. Caracterizada por um aumento rápido, intenso e inapropriado da pressão arterial, a crise hipertensiva é uma das complicações da hipertensão arterial ${ }^{2,4-8}$ associada a uma predisposição genética ou a fatores de risco, como estresse, ingestão excessiva de sal, álcool, tabagismo, obesidade, sedentarismo, doença renal, entre outros distúrbios ${ }^{9}$.

Cirurgiões-dentistas demonstram não ter segurança suficiente para agir corretamente em situações de crises hipertensivas, por isso, o presente estudo tem como objetivo reunir informações sobre a crise hipertensiva, diferenciando as emergências hipertensivas das urgências hipertensivas, de modo a colaborar para o melhor entendimento sobre 0 tema pelos cirurgiões-dentistas que ainda têm insegurança quanto às emergências médicas. 


\section{Revisão de literatura}

\section{Patogênese da crise hipertensiva}

$\mathrm{Na}$ maioria dos casos, as principais causas da crise hipertensiva são o controle inadequado da pressão arterial, a não adesão à terapia anti-hipertensiva ou o abandono do tratamento ${ }^{5}$. Geralmente, a pressão arterial diastólica eleva-se para uma média de $120 \mathrm{mmHg}^{2,10}$. Ansiedade, mal-estar, tontura, falta de ar, alterações visuais, cefaleia severa, tosse, dor no peito e vasoespasmos são alguns dos sinais e sintomas que surgem com o aumento da pressão arterial, apesar de serem bastante inespecíficos, variando de caso para caso ${ }^{2}$.

As crises hipertensivas ocorrem devido a um desequilíbrio entre o débito cardíaco e a resistência vascular periférica ${ }^{5}$. O aumento na pressão arterial sobrecarrega os vasos sanguíneos e o coração, requerendo rápida e correta correção, especialmente em pacientes com outras patologias ${ }^{3}$. Essa sobrecarga provoca lesões endoteliais e necrose fibrinoide das arteríolas, que gera uma deposição de plaquetas e de fibrina e, consequentemente, a quebra da autorregulação normal do fluxo sanguíneo. Com isso, a isquemia estimula a liberação de substâncias vasoativas, gerando um círculo vicioso $0^{5}$.

\section{Prevalência}

No Brasil, a prevalência média da hipertensão arterial sistêmica varia de $24,8 \%$ a $44,4 \%$, conforme estudos feitos com a população local de diversos lugares. A média mundial é de $26,4 \%$. É pequena a diferença da prevalência entre homens e mulheres, porém a prevalência em indivíduos da raça branca é duas vezes menor que em pessoas de outras raças ${ }^{11}$.

Em relação à prevalência e à incidência da crise hipertensiva propriamente dita, são poucos os estudos encontrados na literatura. Porém, nos estudos disponíveis estima-se que aproximadamente apenas $1 \%$ dos hipertensos apresenta crise hipertensiva, tendo como principais fatores de risco o tabagismo e o diabetes ${ }^{6,12}$.

Em um estudo de doze meses de duração, realizado em 23 unidades básicas de saúde de um município da região Sul do Brasil, 422 pessoas foram diagnosticadas com hipertensão arterial sistêmica. Dentre elas, $50 \%$ afirmaram que não aderiram à farmacoterapia, e $12 \%$ foram hospitalizadas por complicação da hipertensão, sendo que $47 \%$ por crise hipertensiva ${ }^{1}$. Em João Pessoa, na Paraíba, 12\% dos atendimentos de emergência feitos pela Samu a idosos foram devido a crises hipertensivas ${ }^{13}$.

Ao longo do ano 2009, Pinna et al. ${ }^{14}$ realizaram um estudo em dez hospitais da Itália e constataram que cinco de cada mil pessoas passaram pela experiência de crise hipertensiva. Nesse estudo, foram relatados 1.546 casos de crise hipertensiva, sendo que $23 \%$ dos pacientes não sabiam que sofriam de hipertensão arterial.

\section{Urgência e emergência hipertensiva}

A crise hipertensiva pode ou não estar associada a lesões de órgãos-alvo, diferenciando as emergências hipertensivas das urgências hipertensivas ${ }^{2,4,5,14,15}$. Pacientes que passaram por uma situação de urgência ou emergência hipertensiva têm maior chance de desenvolver outras patologias em um período de dois anos, tais como síndromes isquêmicas agudas, acidente vascular encefálico, fibrilação atrial, edema agudo de pulmão e dissecção aórtica ${ }^{4}$.

As emergências hipertensivas são basicamente a elevação inapropriada da pressão arterial, podendo gerar um quadro clínico grave e até risco de morte. Nesse tipo de situação, ocorrem lesões nos órgãos-alvo, que podem ser neurológicas, miocárdicas, vasculares, hematológicas ou renais ${ }^{8,15-17}$. As lesões mais frequentes nas emergências hipertensivas são a dissecção da aorta, a encefalopatia hipertensiva, o infarto ou hemorragia cerebral, o infarto do miocárdio, a angina instável ou edema agudo do pulmão, a insuficiência renal e a pré-eclâmpsia ou eclâmpsia ${ }^{7}$.

É importante destacar que a gravidade da situação não é determinada pelo valor final da pressão arterial, mas sim pelo quanto ela aumentou ${ }^{5}$. Além disso, a velocidade de aumento da pressão arterial é um importante fator para quantificar o dano vascular ${ }^{15}$.

Em caso de emergência hipertensiva, ressalta-se a necessidade de hospitalização do paciente a fim de baixar os valores tensionais em minutos ou horas? Nesses casos, geralmente, o controle da pressão arterial é feito com a administração de fármacos via parenteral, geralmente endovenosa, e com observação intensa em unidade de terapia ${ }^{5}$. Esses procedimentos visam à reversão do dano ao órgão-alvo, e não ao retorno dos níveis normais da pressão arterial $^{15}$.

Nas urgências hipertensivas, a pressão arterial também sofre um aumento acentuado, porém não há lesões de órgãos-alvo e, portanto, não há risco de morte iminente. Nesses casos, os valores da pressão arterial devem ser reduzidos gradativamente em um período de 24 a 48 horas, geralmente por meio de medicação oral e sem internação hospitalar do paciente. Ainda assim, o paciente precisa ficar por duas horas sob observação clínica em um ambiente calmo e de baixa luminosidade, pois isso colabora com o diagnóstico diferencial de pseudocrise hipertensiva, na qual o repouso pode resolver os sinais e os sintomas da crise. Medidas como essa podem reduzir os níveis de pressão arterial sem a administração de anti-hipertensivos ${ }^{5,8,15-17}$.

Para urgências hipertensivas, pode-se fazer o uso do captopril. Esse fármaco é um eficaz inibidor da enzima de conversão da angiotensina (IECA), sua absorção é rápida e apresenta boa tolerabilida- 
de. A recomendação para doses iniciais é de $25 \mathrm{mg}$ por via sublingual ou oral, podendo ser repetidas em intervalo de quarenta minutos a uma hora ${ }^{5}$.

É importante ressaltar que se deve evitar a promoção de quedas excessivas na pressão arterial, visto que essas quedas podem causar isquemia renal, cerebral ou coronariana ${ }^{5}$.

Existem ainda as pseudocrises hipertensivas, que também são elevações acentuadas da pressão arterial, porém sem risco potencial, sem evidências de lesão aguda de órgãos-alvo e sem risco iminente de morte. Isoladamente, ou seja, fora do quadro clínico de crises hipertensivas, o aumento da pressão arterial dificilmente requer terapia específica ${ }^{18}$.

\section{Conduta}

O profissional que se depara com esse tipo de situação deve estar apto a reconhecer as manifestações da crise e definir prontamente o tratamento, a fim de reduzir a morbidade e a mortalidade associadas a essas complicações ${ }^{15}$. Assim, o domínio da execução não é específico de apenas um tipo de profissional, mas sim de todos os profissionais que possam se deparar com essas situações clínicas. Em um estudo realizado na Bahia por Sobrinho et al. ${ }^{18}$ (2007), médicos trataram quase todos os casos de pseudocrise hipertensiva como crise verdadeira, nesse caso é importante ressaltar que a redução brusca da pressão arterial poder transformar uma pseudocrise em crise verdadeira. Segundo Lúcio e Barreto ${ }^{9}$, "[...] a maioria dos profissionais de odontologia não se sente segura e capacitada para o correto atendimento de uma emergência, ficando assim dependente da presença médica para socorrer o paciente".

É imprescindível que o cirurgião-dentista saiba como proceder para prevenir uma crise hipertensiva, pois o atendimento odontológico está intimamente ligado ao medo, que promove aumento da ansiedade e, por conseguinte, do estresse. Para minimizá-lo, o profissional deve optar por fazer consultas curtas e preferencialmente no período da manhã, controlar eventos de dor, conversar e distrair o paciente e, quando necessário, indicar o uso de ansiolíticos. Quando o procedimento precisar de anestesia, o profissional deve dar preferência às soluções anestésicas que contenham felipressina como vasoconstritor ${ }^{9}$.

Previamente ao atendimento odontológico, é de grande importância que se faça uma avaliação de saúde geral do paciente e também de seu estado emocional. Pela anamnese, o profissional terá boas condições para realizar um atendimento de forma segura ${ }^{9}$. A definição do melhor tratamento deve ser baseada em um consenso das informações do paciente previamente obtidas, devendo-se considerar a etiologia da hipertensão, a extensão das lesões de órgãos-alvo e o perfil hemodinâmico individual do paciente $^{15}$.
Quando o paciente apresentar sinais e sintomas característicos de uma crise hipertensiva, como elevação da pressão arterial, cefaleia, epistaxe, hemorragia gengival após manipulação, tontura, mal-estar, confusão mental e distúrbios visuais, o atendimento deve ser interrompido imediatamente. O paciente deve ser colocado em posição confortável e seus sinais vitais devem ser monitorados. $\mathrm{O}$ profissional deve administrar captopril, na dose de $25 \mathrm{mg}$ a $50 \mathrm{mg}$, por via sublingual. Uma vez controlada a crise, o paciente deve ser encaminhado para avaliação médica o quanto antes ${ }^{9}$.

\section{Considerações finais}

É de extrema importância que o cirurgião-dentista seja devidamente treinado para realizar o suporte básico de vida. As crises hipertensivas podem ocorrer a qualquer momento, em qualquer lugar e com qualquer paciente, e devem ser bem diagnosticadas para que se faça o adequado tratamento. Na odontologia, os profissionais preocupam-se muito com técnicas odontológicas, equipamentos e atualizações, mas acabam esquecendo o principal ponto de sua formação acadêmica: a saúde e a vida do seu paciente.

\section{Abstract}

Objective: This study aims to gather information about hypertensive crisis, to differentiate hypertensive emergency from hypertensive urgency, to present procedures for dentists, and to contribute for the better understanding of the subject by dentists who feel uncertain about medical emergencies. Literature review: Characterized by a fast, intense, and improper increase in blood pressure, hypertensive crisis is one of the complications of arterial hypertension. It may occur due to an imbalance between cardiac output and peripheral vascular resistance. The increase in blood pressure overloads the blood vessels and the heart, requiring fast and accurate correction, especially in patients with other pathologies. Hypertensive crises may or may not be associated with target-organs damage, which distinguishes hypertensive emergencies and hypertensive urgencies. Final considerations: It is essential that dentists know how to prevent a hypertensive crisis since dental care is closely linked to fear, which promotes an increase in anxiety and therefore in stress.

Keywords: Hypertension. Emergency treatment. Dentistry.

\section{Referências}

1. Barreto MS, Marcon SS. Hospitalização por agravos da hipertensão arterial em pacientes da atenção primária. Acta Paul Enferm 2013; 26(4):313-7.

2. Maciel GDSL, Guedes HM. Crise hipertensiva no atendimento de urgência e emergência. Rev Enferm Int 2009; 2(1):238-46. 
3. Miguel Júnior A, Reverberi CA. Urgência e emergência na hipertensão arterial. Rev Ciênc Med PUCCAMP 1992; 1(2):54-7.

4. Silva MAM, Rivera IR, Santos ACS, Barbosa CF, Oliveira Júnior CAS. Crise hipertensiva, pseudocrise hipertensiva e elevação sintomática da pressão arterial. Rev Bras Cardiol 2013; 26(5):329-36.

5. Martin JFV, Loureiro AAC, Cipullo JP. Crise hipertensiva: atualização clínico-terapêutica. Arq Ciênc Saúde 2004; 11(4):253-61.

6. Martin JFV, Higashiama E, Garcia E, Luizon MR, Cipullo JP. Perfil de crise hipertensiva. Prevalência e apresentação clínica. Arq Bras Cardiol 2004; 83(2):125-30.

7. Santos M, Rodrigues T. A hipertensão arterial na urgência. Rev Port Clin Geral 2008; 24:411-7.

8. Gasques JCP, Roland DMS, Cesarind CB. Caracterização da crise hipertensiva em pacientes de grupo de hipertensão de um ambulatório-escola. Rev Enferm UERJ 2008; 16(1):46-50.

9. Lúcio PSC, Barreto RC. Emergências médicas no consultório odontológico e a (in)segurança dos profissionais. Rev Bras Ciênc Saúde 2012; 16(2):267-72.

10. Tavares A, Kohlmann Júnior O. Tratamento da crise hipertensiva. HiperAtivo 1998; 5(2):120-5.

11. Cesarino CB, Cipullo JP, Martin JFV, Ciorlia LA, Godoy MRP, Cordeiro JA et al. Prevalência e fatores sociodemográficos em hipertensos de São José do Rio Preto - SP. Arq Bras Cardiol 2008; 91(1):31-5.

12. García GM, Miúdo V, Alves CG, Lopes M, Gomes JV. Caracterização dos pacientes com menos de 46 anos internados com emergência hipertensiva no Hospital do Prenda. Rev Port Cardiol 2014; 33(1):19-25.

13. Barros MAA, Oliveira DST, Carvalho MAP, Fernandes MGM, Costa KNFM, Santos KFO. Características dos agravos e da assistência prestada aos idosos em um serviço pré-hospitalar móvel. Rev Enferm UERJ 2013; 21(Esp. 1):569-74.

14. Pinna G, Pascale C, Fornengo P, Arras S, Piras C, Panzarasa $\mathrm{P}$ et al. Hospital admissions for hypertensive crisis in the emergency departments: a large multicenter Italian study. Plos One 2014; 9(Iss 4):1-6.

15. Cardoso ME. Emergências hipertensivas. Rev Hupe 2013; 12(3):66-77.

16. Franco RJS. Crise hipertensiva: definição, epidemiologia e abordagem diagnóstica. Rev Bras Hipertens 2002; 9(4):340-5.

17. Feitosa-Filho GS, Lopes RD, Poppi NT, Guimarães HP. Emergências hipertensivas. Rev Bras Ter Intensiva 2008; 20(3):305-12

18. Sobrinho S, Correia LCL, Cruz C, Santiago M, Paim AC, Meireles B et al. Ocorrência e preditores clínicos de pseudocrise hipertensiva no atendimento de emergência. Arq Bras Cardiol 2007; 88(5):579-84.

Endereço para correspondência:
Júllian Dalla Libera Pegoraro
Rua 14 de Julho, 32
95300-000 Lagoa Vermelha, RS
Telefone: (51)9676-6427
E-mail: pegorarojdl@gmail.com

Recebido: 01/07/14. Aceito: 22/09/15. 